\title{
Induction of cardiomyocyte proliferation, a new way forward for true myocardial regeneration?
}

\author{
Zhiyong Lei $^{1 \#}$, Marijn Peters ${ }^{1,2 \#}$, Joost P. G. Sluijter ${ }^{1,2}$ \\ ${ }^{1}$ Department of Cardiology, Laboratory of Experimental Cardiology, University Medical Center Utrecht, University Utrecht, Utrecht, the \\ Netherlands; ${ }^{2}$ UMC Utrecht Regenerative Medicine Center, University Medical Center Utrecht, the Netherlands \\ "These authors contributed equally to this work. \\ Correspondence to: Joost P. G. Sluijter, PhD. Department of Cardiology, Experimental Cardiology Laboratory, Division Heart and Lungs, University \\ Medical Center Utrecht, Heidelberglaan 100, room G02.523, 3584 CX Utrecht, the Netherlands. Email: j.sluijter@umcutrecht.nl. \\ Provenance: This is an invited Editorial commissioned by the Section Editor Dr. Jin Li (Cardiac Regeneration and Ageing Lab, School of Life \\ Sciences, Shanghai University, Shanghai, China). \\ Comment on: Huang W, Feng Y, Liang J, et al. Loss of microRNA-128 promotes cardiomyocyte proliferation and heart regeneration. Nat Commun \\ 2018;9:700.
}

Received: 18 July 2018; Accepted: 19 July 2018; Published: 01 August 2018.

doi: $10.21037 /$ ncri.2018.07.04

View this article at: http://dx.doi.org/10.21037/ncri.2018.07.04

\section{Heart failure and current therapies}

Ischemic heart disease (IHD) and heart failure (HF) are major causes of morbidity and mortality worldwide and although acute treatment during myocardial infarction has improved significantly, chronic burden of the disease gained much attention recent years $(1,2)$. The major cause of the failing hearts, however, the loss of contractile cardiomyocytes is currently still a problem to overcome. The only treatment modalities that are nowadays available for contractile dysfunction includes left ventricle assist devices (LVAD) and heart transplantation. Both are well accepted therapies but with different draw-backs for large patients groups, thereby pointing to the lack of enough donor hearts and the need for endured medication use, respectively (3).

Regenerative medicine is a direction in which the loss of contractile tissue is aimed to be replaced by the exogenous addition of progenitor or stem cells. Although originally having high expectations, which was implicated by scientific progress in preclinical models, and long-term, randomized clinical trials with good safety profiles for delivery of cells to the myocardium, regenerative therapy for cardiovascular disease had been inconsistent and shown modest efficacy thus far. Recent reviews have nicely illustrated current drawback and the need for improvements for these strategies, using cells or cell-derived products (4-6). Due to the lack of major steps forward other directions have been explored, boosted by the observations of Bergmann and his colleagues that cardiomyocytes from the human heart have a certain degree of renewal capacity (7). This is combination with the work of Poss, which demonstrated the potential of cardiomyocytes to divide, has pushed new regenerative strategies forward (8).

\section{Cardiomyocytes renewal and the cell cycle}

The cardiac regenerative capacity mainly depends on the ability of cardiomyocytes to proliferate after injury $(9,10)$. Adult amphibian hearts can completely regenerate after apex resection through cell cycle re-entry of pre-existing cardiomyocytes in the infarct borderzone $(9,11)$. In the mammalian heart, cardiomyocytes proliferate prenatally and withdraw from the cell cycle 7 days after birth (12). At 3 days after birth, cardiomyocytes retain a constant cell volume and are primarily mononucleated (13). This is in contrast to the abundance of multinucleated cardiomyocytes through DNA replication in the absence of cytokinesis 1-2 weeks after birth $(13,14)$. Under basal conditions in the adult mammalian heart, proliferation of cardiomyocyte replaces the cardiomyocytes that die due to apoptosis at a rate of $0.5-1 \%$ a year (7). Although after cardiac injury the cardiomyocyte proliferation rate increases, it remains 
insufficient to regenerate the heart (15). The endogenous repair mechanisms that occur after injury in the neonatal and the amphibian heart do lead to complete restoration of the myocardium. Stimulating the same endogenous repair mechanism in the adult mammalian heart, through the proliferation of pre-existing cardiomyocytes, can currently be considered the most feasible method to regenerate the heart in a clinical setting. The program that regulates cardiomyocyte cell cycle withdrawal remains largely unknown but is hypothesized to result from the relative hypoxia and the postnatal metabolic shift from anaerobic glycolysis to oxidative phosphorylation (16). This is in line with studies demonstrating that in amphibian hearts, cardiac regeneration depends on hypoxia signaling (17). Furthermore, the rare population of cycling cardiomyocytes in the adult mammalian heart were found to be located in hypoxic niches (18). Even though it might seem contradictory to the role of hypoxia in regeneration, the infarcted myocardium requires sufficient revascularization for functional regeneration to occur. The neonatal and zebrafish heart are heavily vascularized after cardiac injury and without vascularisation of the infarct area, regeneration ends prematurely $(12,19)$.

To investigate the regulators of cardiac regeneration studies have analyzed the changes in expression of coding and the non-coding genes at $\mathrm{p} 7$ when the mammalian heart loses is capacity to regenerate. Analysis of the non-coding transcriptome in cardiac disease indicated the essential role of non-coding RNAs in cardiac biology and in epigenetic control of gene expression in the diseased heart $(20,21)$. miRNAs regulate the expression of a target mRNA by binding to the 3'UTR and hereby either preventing the translation of the mRNA or aiding in its degradation. LncRNAs (>200 nucleotides) can regulate epigenetic gene silencing, bind to miRNAs, and function as protein scaffolds (22). Exploring the expression at P1, P3, P5, $\mathrm{P} 7$, and P10 showed differential expression of the 413 miRNAs and 545 lncRNAs in both endothelial cells and cardiomyocytes (23). A marked transition between P3 and P5 was found of 240 miRNAs including the increase in expression of miR-451, miR-195, and miR22 and a decrease in expression of miR-6240. miR-451 was previously reported to protect erythrocytes against oxidative stress which could also be a potential explanation of its upregulation in cardiac development towards relative hyperoxia (24). miR-195 is member of the miR-15 family which has been described to be upregulated in the postnatal mammalian heart and contribute to postnatal cardiomyocyte cell cycle withdrawal (25). miR-22 induces cardiac senescence by preventing cardiomyocyte proliferation and by activating cardiac fibroblasts (26). Other miRNAs that have been associated with the induction of cardiomyocyte proliferation include miR-590, miR199a (27), miR-210 (28), and miR-133 (29). Recent evidence identified 96 miRNAs to be able to induce cardiomyocyte proliferation in human induced pluripotent stem cell derived cardiomyocytes (30). Sixty-seven of these miRNAs targeted the Hippo pathway through the repression of Hippo and the activation of Yap, suggesting this pathway might be a promising target for cardiac regenerative therapy.

\section{miRNA-128, a new player that promotes cardiomyocyte proliferation}

Recently in Nature Communications, Huang and colleagues reported a new player, microRNA-128 (miRNA-128), which is upregulated in cardiomyocytes during the postnatal switch from proliferation to terminal differentiation (31). miR-128 is an intronic miRNA, encoded by two isoforms; miR-128-1 and miR-128-2 (32). The pri-miR-128-1 gene resides within the $\mathrm{R} 3 \mathrm{H}$ domain containing protein 1 gene (R3HDM1) and pri-miR-128-2 lies within the cAMP-regulated phosphoprotein, $21 \mathrm{kDa}$ gene (ARPP21, also known as regulator of calmodulin signaling, RCS). This organization is conserved in human, rat, and mouse genomes (32). Early studies pointed to a role of miRNA-128 as a tumor suppressor, but others found a role in neural and brain development and in behavior disorders (33-35). In the myocardium, however, the expression of miR-128 is low during early developmental stages but is increased during postnatal heart growth.

The cardiac-specific overexpression of miR-128, via a tet-off transgenic mouse system, impaired cardiac homeostasis by inducing a progressive heart mass and thereby to increased heart-to-body weight ratios. Detailed analyses suggested that cardiomyocytes increased in size, leading to a hypertrophic response and thereby to a reduced fractional shortening and ejection fraction. More detailed analyses displayed that cardiomyocyte proliferation was reduced upon overexpression of miR-128, with no effect on apoptosis rates, thereby suggesting an early cell cycle exit for cardiomyocytes. Interestingly, the cardiac-specific deletion of miR-128 in both in vitro cultured neonatal cardiomyocytes as in vivo mouse hearts resulted in an increased dedifferentiation of cardiomyocytes and increased proliferation rates. Interestingly, although the number of 
cardiomyocytes was larger, being smaller in size, the mice developed normally and exhibited no cardiac dysfunctions. Confirmatory, the overexpression of miR-128 in neonatal mice at $\mathrm{P} 1$ inhibited cardiac regeneration upon myocardial apex resection, whereas deletion of miR-128 in adult mice promoted cardiac regeneration upon ischemic injury. The authors further explored putative targets and suggest a role for Suz12, thereby affecting histone modifications and p27, and promoting cardiomyocytes into the S-phase to divide.

\section{Potential impact and future directions}

miR-128 was previous being reported as a neuronalenriched miRNA, but also involved in cardiac repair of lower vertebrates such as the newt (36). In these species, however, the effects observed where mainly attributed to proliferation in the non-cardiomyocyte populations and on extracellular matrix deposition in which inhibition of miR-128 led to these effects. Although the authors suggest this might be due to differences in complex of the heart cell types between the species, it also points towards some concerns for translation of these findings regarding miRNA therapeutics for patient care if we cannot control potential off-target cell effects (31).

In the past few years, several types of miRNA inhibitors have been developed such as microRNA antagomiRs, antimiRs and LNAs which have been shown to be very effective in inhibiting their specific targets. We and many others labs have shown that these microRNA inhibitors demonstrate much longer effects than conventional pharmaceutics (37). This long-lasting effect will definitely increase the possibility of side-effect, especially in this case of miR-128, which is a potent cell cycle regulator with known effects in other cell types and organs. For these reasons, there is a strong need to develop new methods to increase miRNA therapeutic delivery, prevent offtarget cellular uptake and, in the case of miR-128, increase cardiac-specific uptake. Our lab has further developed a local delivery technology called ultrasound-triggered microbubble destruction (UTMD) for microRNA delivery. In this technology, positive charged gas-filled microbubbles can be loaded with negative charged microRNA inhibitors, antimiR or antagomirs. By locally applying ultrasound, gasfilled microbubbles can be destructed and thereby deliver their cargo to the targeted area (38). Using this technology, we have been able to significantly increase local delivery of antagomir to the healthy heart with minimum sideeffects. Interestingly, similar results were also observed in a myocardial infarction and reperfusion model, even without ultrasound treatment. This is probably caused by the fact that the inhibitors can be enriched in the infarcted region through local injury-induced vascular leakage, which is a possible mechanism how ultrasound triggered microbubble destruction delivers its cargo too (39). Another possible strategy to achieve targeted delivery will be nanovectors which are armed with cardiac homing peptide. Inspired by the observation that post myocardial infarction AT1 levels are increased, Xue et al. have developed a nanovector with AT1 targeting peptides on the surface. Armed with miR-1 inhibitors, this nanovector can significantly inhibit miR-1 induced apoptosis in the infarcted region (40). Although only a few cases have been successful to specific delivery miRNAs into the myocardium, we believe with the continuous efforts to develop new delivery vehicles such as polymers, lipid nanoparticles, and extracellular vesicles with difference formulations in combination with difference targeting strategies, it is possible to take full advantage of microRNA therapeutics while minimize their potential side-effect.

\section{Conclusions}

The growing burden of chronically diseased cardiovascular patients that develop HF needs new treatment modalities to restore contractile properties of the myocardium. Although attempts to minimize the damage to the cardiac tissue in acute settings is successful, still new approaches are needed. Cardiac regenerative medicine offers potential inspiring strategies, including the use of endogenous or exogenous injection of progenitor cell population. Since the simple injection of cell types is hampered by retention of high cell numbers, other options are explored as well, including the stimulation of post-natal cardiomyocyte proliferation. These novel approaches are extremely exciting, especially by the use of miRNA therapeutics, which can lead to longlasting and potent effects. The future directions of these approaches are aiming to further explore the potential of the identified molecules and improve local targeting and timed exposure of the therapeutics to reduce costs and toxic effects and, maybe of most importance for the stimulation of cell division, prevent off-target effects in other cell types.

\section{Acknowledgements}

Funding: This work was supported by Horizon2020 ERC2016-COG EVICARE (725229), Technobeat (668724), 
and the Netherlands CardioVascular Research Initiative (CVON), PPS grant: TOP-EVs (the Nederland Heart Foundation), CAS-NWO Program: Cardiac cell therapy in a preclinical animal model for hereditary cardiomyopathy the Dutch Heart Foundation, Dutch Federations of University Medical Centers, the Netherlands Organization for Health Research and Development, and the Royal Netherlands Academy of Sciences (REMAIN).

\section{Footnote}

Conflicts of Interest: The authors have no conflicts of interest to declare.

\section{References}

1. Townsend N, Wilson L, Bhatnagar P, et al. Cardiovascular disease in Europe: epidemiological update 2016. Eur Heart J 2016;37:3232-45.

2. Writing Group M, Mozaffarian D, Benjamin EJ, et al. Executive Summary: Heart Disease and Stroke Statistics--2016 Update: A Report From the American Heart Association. Circulation 2016;133:447-54.

3. Ponikowski P, Voors AA, Anker SD, et al. 2016 ESC Guidelines for the diagnosis and treatment of acute and chronic heart failure: The Task Force for the diagnosis and treatment of acute and chronic heart failure of the European Society of Cardiology (ESC)Developed with the special contribution of the Heart Failure Association (HFA) of the ESC. Eur Heart J 2016;37:2129-200.

4. Madonna R, Van Laake LW, Davidson SM, et al. Position Paper of the European Society of Cardiology Working Group Cellular Biology of the Heart: cell-based therapies for myocardial repair and regeneration in ischemic heart disease and heart failure. Eur Heart J 2016;37:1789-98.

5. Fernandez-Aviles F, Sanz-Ruiz R, Climent AM, et al. Global position paper on cardiovascular regenerative medicine. Eur Heart J 2017;38:2532-46.

6. Sluijter JPG, Davidson SM, Boulanger CM, et al. Extracellular vesicles in diagnostics and therapy of the ischaemic heart: Position Paper from the Working Group on Cellular Biology of the Heart of the European Society of Cardiology. Cardiovasc Res 2018;114:19-34.

7. Bergmann O, Bhardwaj RD, Bernard S, et al. Evidence for cardiomyocyte renewal in humans. Science 2009;324:98-102.

8. Tzahor E, Poss KD. Cardiac regeneration strategies: Staying young at heart. Science 2017;356:1035-9.
9. Jopling C, Sleep E, Raya M, et al. Zebrafish heart regeneration occurs by cardiomyocyte dedifferentiation and proliferation. Nature 2010;464:606-9.

10. Aguirre A, Sancho-Martinez I, Izpisua Belmonte JC. Reprogramming toward heart regeneration: stem cells and beyond. Cell Stem Cell 2013;12:275-84.

11. Poss KD, Wilson LG, Keating MT. Heart regeneration in zebrafish. Science 2002;298:2188-90.

12. Porrello ER, Mahmoud AI, Simpson E, et al. Transient regenerative potential of the neonatal mouse heart. Science 2011;331:1078-80.

13. Li F, Wang X, Capasso JM, et al. Rapid transition of cardiac myocytes from hyperplasia to hypertrophy during postnatal development. J Mol Cell Cardiol 1996;28:1737-46.

14. Ahuja P, Sdek P, MacLellan WR. Cardiac myocyte cell cycle control in development, disease, and regeneration. Physiol Rev 2007;87:521-44.

15. D'Uva G, Aharonov A, Lauriola M, et al. ERBB2 triggers mammalian heart regeneration by promoting cardiomyocyte dedifferentiation and proliferation. Nat Cell Biol 2015;17:627-38.

16. Puente BN, Kimura W, Muralidhar SA, et al. The oxygenrich postnatal environment induces cardiomyocyte cell-cycle arrest through DNA damage response. Cell 2014;157:565-79.

17. Jopling C, Sune G, Faucherre A, et al. Hypoxia induces myocardial regeneration in zebrafish. Circulation 2012;126:3017-27.

18. Kimura W, Xiao F, Canseco DC, et al. Hypoxia fate mapping identifies cycling cardiomyocytes in the adult heart. Nature 2015;523:226-30.

19. Lepilina A, Coon AN, Kikuchi K, et al. A dynamic epicardial injury response supports progenitor cell activity during zebrafish heart regeneration. Cell 2006;127:607-19.

20. Grote P, Wittler L, Hendrix D, et al. The tissue-specific lncRNA Fendrr is an essential regulator of heart and body wall development in the mouse. Dev cell 2013;24:206-14.

21. Archer K, Broskova Z, Bayoumi AS, et al. Long noncoding RNAs as master regulators in cardiovascular diseases. Int J Mol Sci 2015;16:23651-67.

22. Juni RP, Abreu RC, da Costa Martins PA. Regulation of microvascularization in heart failure-an endothelial cell, non-coding RNAs and exosome liaison. Noncoding RNA Res 2017;2:45-55.

23. Adamowicz M, Morgan CC, Haubner BJ, et al. Functionally conserved noncoding regulators of cardiomyocyte proliferation and regeneration in mouse and human. Circ Genom Precis Med 2018;11:e01805. 
24. Yu D, dos Santos CO, Zhao G, et al. miR-451 protects

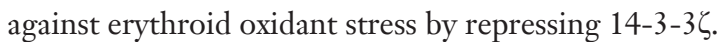
Genes Dev 2010;24:1620-33.

25. Porrello ER, Mahmoud AI, Simpson E, et al. Regulation of neonatal and adult mammalian heart regeneration by the miR-15 family. Proc Natl Acad Sci U S A 2013;110:187-92.

26. Jazbutyte V, Fiedler J, Kneitz S, et al. MicroRNA-22 increases senescence and activates cardiac fibroblasts in the aging heart. Age 2013;35:747-62.

27. Eulalio A, Mano M, Dal Ferro M, et al. Functional screening identifies miRNAs inducing cardiac regeneration. Nature 2012;492:376-81.

28. Arif M, Pandey R, Alam P, et al. MicroRNA-210-mediated proliferation, survival, and angiogenesis promote cardiac repair post myocardial infarction in rodents. $\mathrm{J}$ Mol Med (Berl) 2017;95:1369-85.

29. Yin VP, Lepilina A, Smith A, et al. Regulation of zebrafish heart regeneration by miR-133. Dev Biol 2012;365:319-27.

30. Diez-Cunado M, Wei K, Bushway PJ, et al. miRNAs that Induce Human Cardiomyocyte Proliferation Converge on the Hippo Pathway. Cell Rep 2018;23:2168-74.

31. Huang W, Feng Y, Liang J, et al. Loss of microRNA-128 promotes cardiomyocyte proliferation and heart regeneration. Nat Commun 2018;9:700.

32. Megraw M, Sethupathy P, Gumireddy K, et al. Isoform specific gene auto-regulation via miRNAs: a case study on miR-128b and ARPP-21. Theoretical Chemistry Accounts 2010;125:593-8.

doi: $10.21037 /$ ncri.2018.07.04

Cite this article as: Lei Z, Peters M, Sluijter JP. Induction of cardiomyocyte proliferation, a new way forward for true myocardial regeneration? Non-coding RNA Investig 2018;2:46.
33. Weiss GJ, Bemis LT, Nakajima E, et al. EGFR regulation by microRNA in lung cancer: correlation with clinical response and survival to gefitinib and EGFR expression in cell lines. Ann Oncol 2008;19:1053-9.

34. Krichevsky AM, King KS, Donahue CP, et al. A microRNA array reveals extensive regulation of microRNAs during brain development. RNA 2003;9:1274-81.

35. Ching AS, Ahmad-Annuar A. A Perspective on the Role of microRNA-128 Regulation in Mental and Behavioral Disorders. Front Cell Neurosci 2015;9:465.

36. Witman $\mathrm{N}$, Heigwer J, Thaler B, et al. miR-128 regulates non-myocyte hyperplasia, deposition of extracellular matrix and Islet1 expression during newt cardiac regeneration. Dev Biol 2013;383:253-63.

37. Kwekkeboom RF, Lei Z, Doevendans PA, et al. Targeted delivery of miRNA therapeutics for cardiovascular diseases: opportunities and challenges. Clin Sci 2014;127:351-65.

38. Kwekkeboom RF, Lei Z, Bogaards SJ, et al. Ultrasound and microbubble-induced local delivery of MicroRNAbased therapeutics. Ultrasound Med Biol 2015;41:163-76.

39. Kwekkeboom RFJ, Sluijter JPG, van Middelaar BJ, et al. Increased local delivery of antagomir therapeutics to the rodent myocardium using ultrasound and microbubbles. J Control Release 2016;222:18-31.

40. Xue X, Shi X, Dong H, et al. Delivery of microRNA-1 inhibitor by dendrimer-based nanovector: An early targeting therapy for myocardial infarction in mice. Nanomedicine 2018;14:619-31. 